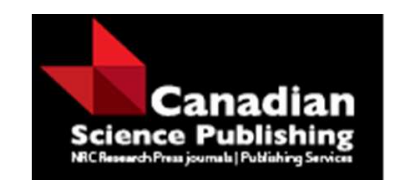

Canadian Journal of Physics

Revue canadienne de physique

\title{
Extreme ultraviolet spectra of highly charged Fe ions in the laboratory versus the excitation of spectra in astrophysical environments
}

\begin{tabular}{|r|l|}
\hline Journal: & Canadian Journal of Physics \\
\hline Manuscript ID & cjp-2016-0623.R2 \\
\hline Manuscript Type: & Review \\
\hline Date Submitted by the Author: & 31 -Oct-2016 \\
\hline Complete List of Authors: & Träbert, Elmar; Ruhr Univ Bochum, Physik und Astronomie \\
\hline Keyword: & $\begin{array}{l}\text { Atomic spectra, Extreme ultraviolet, Beam-foil spectroscopy, Electron beam } \\
\text { ion trap, Lifetime measurement }\end{array}$ \\
\hline \multicolumn{2}{|l}{} \\
\hline
\end{tabular}

\section{SCHOLARONE $^{\text {tw }}$ \\ Manuscripts}




\title{
Extreme ultraviolet spectra of highly charged Fe ions in the laboratory versus the excitation of spectra in astrophysical environments
}

\author{
EImar Träbert
}

\begin{abstract}
The ASOS meetings address atomic spectra and oscillator strengths for astrophysics and laboratory plasmas. Based on examples of Fe spectra from foil-excited ion beams and electron beam ion traps, some practical problems of laboratory studies using these tools in support of astrophysical observations are discussed.
\end{abstract}

Visible and ultraviolet spectra; Atomic excitation and ionization 32.30.Jc; 34.80.Dp

\section{Introduction}

Spectra recorded of the solar corona during eclipses since the middle of the $19^{\text {th }}$ century revealed a number of prominent spectral lines in the visible spectral range that could not be reproduced in the laboratory. One of the suggested solutions to the riddle was a hypothetical element coronium that supposedly existed in the Sun, but was not found on Earth.

In the 1910s and 1920s Manne Siegbahn (at Lund and then at Uppsala (Sweden)) worked in Xray spectroscopy using crystal spectrometers. The measurement of wavelengths in the x-ray range then referred to crystals with layer spacings measured in X-units which had not yet been determined absolutely at the time. Siegbahn designed a grazing-incidence spectrometer with a ruled diffraction grating and tasked his doctoral student Bengt Edlén with trying to link the x-ray crystal measurements to optical wavelengths (which were measured with normal-incidence spectrometers based on ruled gratings). Edlén investigated the properties and offerings of grazing-incidence grating spectrometers thoroughly [1] and, by the 1930s, he became the world expert on the production of multiply ionized atoms and on highly accurate spectroscopy in the extreme ultraviolet (EUV), the wavelength range between $\mathrm{X}$ rays and the vacuum UV (VUV). In my view the EUV wavelength range begins with

Elmar Träbert. ${ }^{1} \quad$ Astronomisches Institut, Fakultät für Physik und Astronomie, Ruhr-Universität Bochum, 44780 Bochum, Germany, and Physics Division, Lawrence Livermore National Laboratory, Livermore, CA 94550, U.S.A.

1 Corresponding author: e-mail: traebert@astro.rub.de 
wavelengths shorter than the transmission cut-off of LiF, that is near $110 \mathrm{~nm}$ (other definitions use the $\mathrm{H} \mathrm{I} \mathrm{Ly}_{\alpha}$ line at $121.5 \mathrm{~nm}$ ); the short-wavelength limit towards soft-x-rays is physically ill defined and may be claimed anywhere in the range from 1 to $10 \mathrm{~nm}$.

Edlén systematically determined wavelengths and studied wavenumber differences for the purpose of establishing atomic energy levels. Following up on a suggestion by astrophysicist Walter Grotrian he confirmed that the wave numbers of prominent corona lines matched term differences in highly charged ions of $\mathrm{Fe}$ (and $\mathrm{Ca}$ ) [2]. This finding rendered the idea of the hypothetical element coronium obsolete, but it implied a new puzzle. While the solar chromosphere features a temperature of about $0.5 \mathrm{eV}$, these highly charged ions can be produced only at energies at least two orders of magnitude higher. Edlén's paper thus caused a paradigm shift about the solar corona and initiated a plethora of attempts to explain how the solar corona could be so hot above a much cooler surface through which the necessary heating energy would have to flow. Direct observations of the solar EUV spectrum became possible only some two decades later, when sounding rockets and spacecrafts carried spectrometers above the Earth's EUV-opaque atmosphere. A particularly successful flight experiment was the Apollo Telescope Mount (ATM) on board the Skylab spacecraft (in the 1970s), finding that the solar EUV spectrum was bright and very rich in lines, offering information for detailed plasma diagnostics. In the decade before, evolving nuclear fusion test experiments had begun to find similarly rich EUV spectra from plasma discharges in the laboratory $[3,4,5]$. Obviously, both light sources warranted detailed study, and it was soon recognized that they profited from each other's analyses for interpretation. It turned out that a plethora of lines from essentially all of the astrophysically abundant elements falls into the EUV wavelength range. Actually, iron contributes the brightest lines to the 17-24 nm wavelength range in the Sun.

The EUV data of elements up to $\mathrm{Fe}(Z=26)$ and $\mathrm{Ni}(Z=28)$ have been studied and evaluated in many laboratories around the world. Brian Fawcett (Culham, England) played a remarkable role in the process, because he also systematized the data with the help of Cowan code computations $[6,7]$. Victor Kaufman, Jack Sugar, and their colleagues at NIST, the JAERI group in Japan, the groups at the observatories of Nice and Meudon (France), Lund (Sweden), and many many others contributed. Even the number of data compilations that attempt to collect and represent the spectroscopic knowledge on the EUV spectrum is too large to cite them appropriately in this short note. I therefore limit myself to two examples. The NIST ASD data collection effort [8] historically is based mostly on laboratory data from, say, vacuum sparks and related discharges as well as plasmas operated at low (tokamak) or high (laser-produced) density, but, of course, it also takes solar data into account. NIST also provides an associated bibliography that yields access to the very many individual contributions to this body of knowledge. In contrast, the CHIANTI project [9] leans towards solar spectra recorded by sounding rockets (SERTS/EUNIS) and spacecrafts (examples are the CDS and SUMER instruments of the longserving joint ESA and NASA Solar and Heliospheric Observatory (SOHO) [10], while one of the latest is Hinode carrying the EIS instrument [11]). Both data bases depend on a legacy of historic data from many different sources, many of which are excellent, but which do not necessarily provide complete coverage and may suffer from calibration inconsistencies. Recently both projects have added calculated wavelength data for some of the spectra (NIST has done so for some light ions, but not for the many-electron ions of Fe; most of the tabulated transition rates anyway are from calculations only). These may be helpful in some situations and misleading in others. Error assessment of the compiled data entries usually requires access to the original data sources and occasionally has surprises with experimental data, while the quest for uncertainty estimates for most computed values regularly runs empty.

This report does not present any new wavelength data. The aim instead is a discussion of certain conditions under which laboratory light sources work and spectra are obtained which one has to be aware of when collecting and interpreting laboratory data which are to be compared to solar data. In the following sections, two spectroscopic tools and techniques are reviewed in their merit for the 
interpretation of the solar EUV spectra of Fe. The first is beam-foil spectroscopy, which began to be employed over 50 years ago $[12,13,14,15,16,17]$ and has by now, in spite of its considerable merits, largely fallen into disuse. The second is the electron beam ion trap, which began its spectroscopic application 30 years ago (see [18] for first-hand reports on the early days of this development) and is still providing important contributions to the field. The report does not attempt to be comprehensive, but reflects certain lines of thought which have guided projects I have been involved in. However, some of the citations to my own work also point to the long lists of references available there, which are not repeated here. The spectroscopic examples discussed are from the wavelength range 20 to $55 \mathrm{~nm}$.

\section{Beam-foil spectroscopy}

L. Kay (in England) and S. Bashkin (in the USA) were nuclear physicists who recognized that something visible to the naked eye might be of interest, even as it was not nuclear physics: they saw that fast ion beams that had passed through thin foil targets (with a thickness of a few hundred atomic layers) might emit visible light. Both were chided by their nuclear colleagues for their diversion, but at least Bashkin stuck with the new field. Needing a spectrometer of sorts, he found interest and equipment with astrophysicist A.B. Meinel. They attached a spectrograph to a nuclear physics scattering chamber and recorded a spectrum which had some lines in the standard locations, but otherwise looked highly unusual. Astrophysical spectra have shown many surprise spectral features, and here Bashkin and Meinel claimed to see similarities with the spectrum of a Nova [13]. In this way beam-foil spectroscopy right from the start established a connection to astrophysics.

One characteristic of the new light source was the number of spectral lines seen in addition to the spectra of classical light sources. Evidently the excitation of higher-lying atomic levels was more efficient in the beam-foil interaction, which was recognized as a consequence of the high electron density at excitation. When the fast ions of the ion beam traverse the target foil, the electron density of the environment is that of solid matter. Collisions with electrons here are much more frequent than most radiative transitions. Hence further collisional excitation competes with radiative de-excitation, and multiply excited levels are easily populated, resulting in a plethora of decay channels (and thus spectral lines). While this may be interesting to some, the richness of the spectra also causes severe problems for the spectral separation of spectral lines of interest. Fortunately, a variation of the ion beam energy influences the charge state distribution, giving researchers a handle for finding out which charge state ion an unknown spectral line might belong to. Also, the ion beam is delivered via a beam transport system with various magnetic filters. Hence the observed emission originates from a single element or even a single isotope. Solar spectra, in contrast, have contributions of all elements and a charge state distribution that varies with the ambient temperature and density of the solar plasma, but these parameters are not under the control of an observer. Next, the solar plasma varies over time (on many time scales), but a faraway observer usually observes with some time integration. The ion beam in the laboratory, however, travels at a velocity of a few percent of the speed of light, and the ion velocity changes little (but some) in the sudden interaction with the exciter foil. The observer looks at the ion beam after it has left the target foil material and travels in vacuum again. Observing the ion beam at selected distances after the foil corresponds to observations at different times after the excitation has ended. For example, at an ion velocity of $1 \mathrm{~cm} / \mathrm{ns}$ (ion beam energy of $0.5 \mathrm{MeV} / \mathrm{amu}$, which is typical for moderate size heavy-ion accelerators) a field of view of $1 \mathrm{~mm}$ width corresponds to a time-of-flight interval of $100 \mathrm{ps}$. If the observation begins at the position of the foil, the first $100 \mathrm{ps}$ of any decay are seen ('prompt' spectra); if the observation of the ion beam begins at a position downstream of the foil, the data will show a later part of the decay history ('delayed' spectra). Depending on the available ion beam current and the dark rate of the detection system, displacements of up to some $15 \mathrm{~cm}$ (time delays up to $15 \mathrm{~ns}$ for the given example) have not been uncommon (displacements up to $8 \mathrm{~m}$ have been tried at the Berkeley Hilac accelerator). Thus it is straightforward to record time-resolved spectra with various start times after excitation (and only fine mechanics, but no fast electronic timing is required). 
Different classes of decays (depending on typical decay rates) dominate the prompt spectra and the delayed spectra, as will be shown below.

In a second mode of operation, with a fixed wavelength setting, data can be recorded as a function of displacement of the exciter foil along the ion beam, across the field-of-view and further upstream, and thus a decay curve of a given spectral feature can be obtained and analyzed in order to derive the radiative lifetime of an atomic excitation level. In this operation, the time resolution is not simply limited by the width of the field-of-view of the detection zone ('window function') of the detection apparatus, but by the knowledge of finer geometry details [19]. The ions of the above example take $10 \mathrm{fs}$ to traverse a foil of $100 \mathrm{~nm}$ thickness. The surface of such a foil may be slightly rough; a typical estimate of the transit time in which an ion experience the change of conditions from high electron density to low density is $10^{-15} \mathrm{~s}$. Electric fields may reach a bit further out from the surface, but this short time range is assumed to determine the coherence of any excitation processes in the ion-foil interaction, and it puts a short-time limit on time-resolution in any beam-foil measurement. In practice, the foil surface roughness, the cross section of the ion beam (several $\mathrm{mm}^{2}$ ), and the detection geometry put the time resolution limit to about $1 \mathrm{ps}\left(10^{-12} \mathrm{~s}\right)$. Practical measurement geometries have enabled outer-shell atomic lifetime measurements from a few picoseconds to many nanoseconds. Yet another option exists for time-resolved measurements on inner-excitation processes. X-rays can escape from the interior of the exciter foil (where the electron density is that of a solid state material), and the foil thickness can be controlled to a few atomic layers. By using foils of different thicknesses, inner-shell processes with time constants on the order of 50 fs have been studied. (More examples of time-resolved beam-foil measurements and references can be found in [16].)

Figure 1 shows EUV spectra of Fe after foil excitation, recorded at the Bochum Dynamitron tandem accelerator. This detail matters, because the specific design of this accelerator lets it cope with rather high ion beam currents (on the order of a few $\mu \mathrm{A}$ particles) from a high-current ion source. A high source current eventually translates into a good signal rate. The spectrum shown was dispersed by a $600 \mathrm{\ell} / \mathrm{mm}$ grating in a $R=2.2 \mathrm{~m}$ grazing incidence monochromator; a channeltron served as the (single-channel) detector. The prompt spectrum (upper part of the figure) is very rich in lines in the range 20 to $40 \mathrm{~nm}$; the overall line intensity pattern drops off towards higher wavelengths, and the spectrum appears to be rather weak in the upper wavelength half. Part of this spectrum shape results from the grating efficiency which decreases from $20 \mathrm{~nm}$ towards longer wavelengths [20], part is due to the observation geometry and atomic physics.

At an ion beam energy of 20 to $24 \mathrm{MeV}$ (as for the data in figure 1), the charge state distribution of $\mathrm{Fe}$ ions after foil excitation has a broad maximum near $\mathrm{Fe}^{13+}$, with $\mathrm{Fe}^{10+}$ to $\mathrm{Fe}^{17+}$ ions expected to contribute notably [21, 22]. In this Fe spectrum, the wavelength range below $40 \mathrm{~nm}$ is dominated by E1-allowed transitions (many of them 3s-3p, 3p-3d, etc.). However, in the first beam-foil spectra of iron group elements obtained at Bochum (Fe below $20 \mathrm{~nm}$ (not shown here), Ni and Cu spectra [23] from some $10 \mathrm{~nm}$ upwards) the line density was so high that because of the many line blends not a single line could be identified from the quite extensive literature. The situation was improved by moving away from observing prompt spectra near the exciter foil and choosing observations somewhat downstream of the foil (delayed spectra) instead. This causes a loss of signal rate which could be afforded only with a particularly suited heavy-ion accelerator as mentioned above. However, the downstream spectrum is cleaner and offers distinct features of immediate interest.

In the line-rich prompt spectra, the transition rates involved yield typical lifetimes shorter than, say, $100 \mathrm{ps}$. The detection system views a decay path corresponding to a time of flight of about $100 \mathrm{ps}$. When set to observe prompt decays, it therefore integrates over the first $100 \mathrm{ps}$ and practically catches most of the available light intensity of each short-lived decay. In contrast, the spin-changing E1 decays of low-lying levels have lower transition rates, with typical associated level lifetimes longer than $1 \mathrm{~ns}$. The same detection system consequently sees only a fraction of such a decay length and thus only a fraction of the available total decay signal. However, the foil can be moved upstream so that the 
observation can follow the decay downstream for the recording of decay curves. The intercombination decay signal, of course, remains low, but the background of short-lived decays dies out faster with distance from the foil than the light intensity of 'slow' decays, and consequently the contrast (signalto-background) may improve. This can be seen in the lower part of figure 1 which has been recorded at a distance from the foil corresponding to a time-of-flight of some $15 \mathrm{~ns}$ after excitation. (Because of fewer blends, the spectrometer slits were set wider in favor of collecting more signal.) This spectrum clearly shows lines, which have been identified with 3s-3p intercombination transitions in Mg-like $\left(\mathrm{Fe}^{14+} /\right.$ spectrum Fe XV), Al-like (Fe $\left.{ }^{13+} / \mathrm{Fe} \mathrm{XIV}\right)$, and Si-like (Fe $\left.{ }^{12+} / \mathrm{Fe} \mathrm{XIII}\right)$ ions of Fe [24, 25]. This case provides a striking example for collaborative values of laboratory and astrophysical work: the intercombination line in the Mg-like ion (Fe XV) was already known in the solar spectrum, but those in the Al- (Fe XIV) and Si- (Fe XIII) like ions had not yet been identified. The beam-foil work guaranteed the single element and an adjustable range of charge states, and the intrinsically timeresolved measurement scheme singled out decays of long-lived levels. At the same time the particular measurement parameters precluded the availability of in-spectrum reference lines, costing a loss of wavelength measurement accuracy. Fortunately, the beam-foil lines were easily matched with suitable unidentified lines in the solar spectrum, and the much better known solar wavelengths could be adopted.

Similar measurements taking advantage of time discrimination have helped with identifying a number of weaker lines in the solar EUV spectrum [26]. Of course, one would like to also employ the foil-excitation process for studies of short-lived levels which contribute to line-dense spectra. Spectral resolution then is of utmost importance, as well as statistically sufficient signal. Directly at the exciter foil, the spectrometer/grating combination used for the Fe spectra in figure 1 cannot sufficiently resolve the Fe spectrum in the wavelength range near $20 \mathrm{~nm}$, because the number of core-excited level decays is so large that the lines merge to a practical continuum (and the Doppler width associated with the observation of fast-moving ions precluded any attempt to reach higher resolution by simply narrowing the spectrometer slits). A higher groove density grating was available, but with that one installed the mechanical drive range of the monochromator did not reach wavelengths beyond $20 \mathrm{~nm}$. However, at wavelengths near $10 \mathrm{~nm}$, using a $3600 \ell / \mathrm{mm}$ grating, the line pattern measured with Ti spectra matched the best corresponding measurements at a tokamak plasma in both resolution and relative line intensities [27]. Unfortunately, the Bochum measurement set-up is no longer available.

\section{Electron beam ion traps}

In the fall of 1986, the electron beam ion traps (EBIT) $[28,29]$ at Livermore (CA, USA) began to be used for spectroscopy (see [18]). In such a device the ions are produced by a high current density electron beam (electron density $n_{e} \approx 10^{11} \mathrm{~cm}^{-3}$ ) striking and ionizing atoms or ions in a low charge state which then are confined by the combined action of a strong magnetic field (then provided by a set of superconducting magnet coils), drift tubes on adjustable electric potentials, and the space charge provided by the electron beam. While in beam-foil spectroscopy the fast ions pass through a stationary target of electrons (electron density $n_{e} \approx 10^{24} \mathrm{~cm}^{-3}$ ), the situation is reversed in an EBIT: the ion cloud in its ultra-high vacuum environment remains stationary and can be bombarded with energetic electrons until the rates of further ionization and losses by recombination and charge exchange match. The charge state distribution (adjustable by the electron beam energy) has an upper limit where the ionization energy exceeds the electron beam energy. SuperEBIT (at Livermore) has produced bare uranium ions, thus demonstrating that all charge states of all elements are accessible [30]. For the ion species and (usually much lower) charge states of interest to astrophysics and most of plasma physics, tabletop versions of EBITs are usually sufficient which nowadays are being built with high-temperature superconducting magnet coils or with permanent magnets.

Spectroscopy on electron beam ion traps began with solid state x-ray detectors (for large solid angle and wide spectral coverage), but soon moved on to much higher spectral resolution instruments for x-rays, EUV, VUV, or visible radiation. For the EUV spectral range discussed above, Livermore 
developed flat-field spectrographs that were mostly equipped with cryogenic CCD cameras. The instruments use no slits; the narrow ion cloud does not exceed the electron beam width (some $70 \mu \mathrm{m}$ ) [31] by much, and this light emission zone then is best imaged directly on to the CCD chip.

A line of moderate resolution spectrographs built at Livermore [32] is based on a $R=5 \mathrm{~m}$ diffraction grating with $1200 \ell / \mathrm{mm}$. These have been employed variously at the Livermore EBITs and other plasma machines, most extensively at the NSTX machine at Princeton Plasma Physics Laboratory. At Livermore, many measurements have addressed the EUV spectra of Fe ions in various charge states. (For examples of studies largely based on those moderate-resolution flat field spectrographs see [33, 34] and further references therein. )

An iron spectrum recorded with such an instrument is shown in figure 2, alongside a beam-foil spectrum (part of the spectrum shown in figure 1). In terms of spectral resolution, the two spectra are rather similar, and the similar shape of the background in the wavelength interval 20 to $30 \mathrm{~nm}$ suggests that both recordings encounter a similar density of unresolved weak emission features. The counting statistics is far superior in the EBIT spectrum, because the multi-channel detector permits data accumulation of each channel for the full exposure time $(20 \mathrm{~min})$, whereas the beam-foil spectrum results from a scanning monochromator with a single-channel detector that collects signal for maybe $20 \mathrm{~s}$ at each instrument setting. The beam-foil spectrum has been recorded at an ion beam energy of $20 \mathrm{MeV}$, which is much lower than the energy for optimum yield of Na-like ions ( $\mathrm{Fe} \mathrm{XVI,} \mathrm{Fe}^{15+}$ ) which would be near $60 \mathrm{MeV}[21,22]$. Nevertheless, the $3 \mathrm{~s}-3 \mathrm{p}_{3 / 2}$ transition $(\lambda=33.5410 \mathrm{~nm})$ appears as the strongest line in this spectrum. (The wavelength values here are taken from the NIST tables [8], which in our cases differ by up to $0.001 \mathrm{~nm}$ from the CHIANTI values [9].) The two strong Fe XVI 3p-3d lines $(\lambda=25.1061 / 26.2976 \mathrm{~nm})$ appear at about the same line intensities as the two 3s-3p transitions $(\lambda=33.5409 / 36.0758 \mathrm{~nm})$ (notwithstanding the efficiency curves of the two instruments, which has not been corrected for), and they feed the latter transitions directly; they are consecutive links of their respective decay chains. The EBIT spectrum has been recorded at an electron beam energy of $600 \mathrm{eV}$. This electron beam energy, converted by the mass ratio of electrons and ions, corresponds to an ion beam energy of $61 \mathrm{MeV}$ in a beam-foil experiment, close to where the Na-like ion charge state would be highest.

Actually, the electron beam energy is higher than the ionization potential of Fe XVI, and under these conditions Na-like ions can be ionized to become Ne-like (Fe XVII), which, however, do not have prominent spectral lines in this wavelength range. The strongest line in this EBIT spectrum is the resonance line $(\lambda=28.4164 \mathrm{~nm})$ of $\mathrm{Mg}$-like Fe XV, a lower charge state ion. In the EBIT spectrum of figure 2 (bottom), at first glance the Fe XVI 3p-3d lines appear stronger than the Fe XVI 3s-3p lines (again, without correction for the grating efficiency). However, the strong lines (with a similar line ratio as in the beam-foil spectrum) close to the Fe XVI 3p-3d line positions actually are the Fe XIV $3 s^{2} 3 p$ - $3 \mathrm{~s} 3 \mathrm{p}^{2}$ transitions at $25.2188 \mathrm{~nm}$ and $26.4785 \mathrm{~nm}$, respectively, whereas the true Fe XVI 3p-3d lines are much weaker in the EBIT spectrum than in the beam-foil one. Evidently the Na-like ion resonance lines (3s-3p) appear much more strongly in the beam-foil spectrum even far below the charge state maximum, because they are fed by equally strong $3 \mathrm{p}-3 \mathrm{~d}$ transitions (and these in turn are cascade-fed by many more high-lying levels), but not so in EBIT where the high-angular momentum levels are not easily reached by electron collision from the ground state. Hence the two spectra of figure 2 reflect different charge state distributions at source, and very different relative level populations as well.

Beyond the appearances of the prominent lines, the spectra differ in the number of weak lines. A fair number of weak to moderate lines appear in the beam-foil spectrum that seem to have no counterpart in the EBIT spectrum. Some of these form line clusters which typically arise from transitions between levels of near-maximum angular momentum for a given principal quantum number $n$ (socalled yrast transitions). Such high-angular momentum levels do not connect by direct E1 transitions to the ground configuration. They are not much populated in EBITs where most of the ions decay to the ground state before the next excitation occurs, but the high-angular momentum levels are reached 
easily via cascades from the plethora of high-lying levels excited in the ion beam - foil interaction. This is a prominent indicator of the electron density dependence of the level excitation. Even more drastically than the above EBIT example of the Fe XVI 3p-3d lines versus the 3s-3p lines, the beamfoil spectrum features two strong lines of the Mg-like ion (Fe XV), whereas the EBIT spectrum shows only one prominently. The $3 \mathrm{~s}^{2}{ }^{1} \mathrm{~S}_{0}-3 \mathrm{~s} 3 \mathrm{p}{ }^{1} \mathrm{P}_{0}^{\mathrm{o}}$ resonance line $(\lambda=28.4164 \mathrm{~nm})$ is seen in both spectra, while the dominant line of the $3 \mathrm{~s} 3 \mathrm{p}^{3} \mathrm{P}^{\mathrm{o}}-3 \mathrm{p}^{2}{ }^{3} \mathrm{P}$ transition multiplet $\left(J=2-J^{\prime}=2, \lambda=30.4894 \mathrm{~nm}\right)$ is hardly notable in the EBIT spectrum. This is typical for EBIT spectra; the electron density is so low that practically all excited levels have time to decay before the next excitation. Consequently almost only the ground states are populated and collisional excitation starts there, ground state transitions predominate, and transitions involving an excited lower level are rarely seen [35].

In the context of astrophysics, the low-density environment in an EBIT is more interesting than the high electron density in the beam-foil interaction. The electron density in an EBIT is closest among terrestrial light sources to at least the (relatively) high-density parts of the corona, the solar flares $\left(n_{e} \approx 10^{11} \mathrm{~cm}^{-3}\right.$ ), while the quiet corona has an electron density on the order of $n_{e} \approx 10^{8} \mathrm{~cm}^{-3}$. (Density and temperature together have a strong influence on the spectra; EBIT spectra at low and high electron beam energies can approximate the spectra of solar quiet zones or solar flares, for an example see [36].) Tokamak plasmas are also considered 'low density', but at $n_{e} \approx 10^{14} \mathrm{~cm}^{-3}$ they are denser than the EBIT conditions, but still close enough for mutual interest. Although the spectra in figure 2 are not resolved well enough to separate and identify all lines, they can play a unique role in the quest for understanding solar coronal plasmas.

Beam-foil spectra have lines of only one element at a time, and EBIT spectra can usually be prepared to be dominated by one element, plus a few lines from light elements that often serve for wavelength calibration. This not-quite-one-element-only feature results from the problem of injecting elements into an EBIT without spoiling the ultrahigh vacuum. In many cases, organometallic compounds (for $\mathrm{Fe}$, for example, ferrocene $\mathrm{C}_{10} \mathrm{H}_{10} \mathrm{Fe}$ or ironpentacarbonyl $\mathrm{Fe}(\mathrm{CO})_{5}$ ) or convenient molecules are used which are easily breaking up under the impact of the energetic electron beam. The heaviest element is most easily ionized and thus best trapped, while light elements evaporating from the trap help to cool the remaining ion cloud. The solar corona features, of course, many elements, and this poses problems when trying to identify unknown lines. Here EBIT spectra, even if only moderately resolved, can support the identification effort. Moreover, the problem of line blends can be reduced, if in a laboratory setting the elemental mix can be varied. For example, the He II line near $30.4 \mathrm{~nm}$ dominates a section of the solar EUV spectrum, and largely unfiltered exposures near this wavelength are sometimes used as a monitor of the full activity of the sun (an example is one of the eight channels of the Solar Dynamics Observatory (SDO) AIA instrument [37]). Out of scientific curiosity a recent set of measurements at the Livermore EBIT has demonstrated spectra of various elements with and without He [38], showing several prominent lines of other elements within the width of the He II line profile. Of course, the brightness of the He II line in the sun outshines all other lines in the particular $S D O / A I A$ instrument channel. However, the Fe spectra of the same data set show that the Fe spectrum is too complex for a full analysis at only moderate resolution (such as in figure 2).

Much higher spectral resolution (up to a resolving power $\lambda / \Delta \lambda \approx 3000$ so far) has been obtained

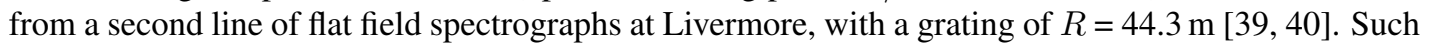
a spectrograph has been employed in a series of measurements in support of the (SDO)/AIA instrument [37] which observes seven narrow wavelength bands in the EUV; in six of these channels Fe lines of ions in specific charge states dominate (the seventh is the aforementioned He II monitor). By correlation of the individual channel signals, the AIA experiment establishes a temperature measurement in the field of view. The individual observation channels are 0.1 to $2 \mathrm{~nm}$ (FWHM) wide in wavelength and comprise various other known lines as well, which the analysis includes via spectral modeling $[41,42]$ based on collections in data bases. The high resolution EBIT measurements are comparable in spectral resolution with the EIS spectrograph onboard the Hinode spacecraft [11]. The laboratory

NRC Canada 
measurements $[36,43,44]$ have detected a fair number of spectral lines not evidently included in those models, and they check on the mutual consistency of the holdings of two major data bases (NIST [8] and CHIANTI [9]) as well. One of those detail studies falls into the wavelength range discussed here [44]. While most of the strong Fe lines apparently are represented consistently in the two data bases, this experiment finds a multitude of unidentified weaker Fe lines which, when taken together, may well amount to as much signal as some of the lines presently included in the spectral model. Thus the models are corroborated overall, but they should benefit from an eventual completion which might affect the results by a few percent while improving the systematic error budget.

\section{Conclusions}

Beam-foil spectroscopy as a technique is fading away after the retirement of two generations of its primary practitioners. The beam-foil interaction also populates levels rarely seen elsewhere, and thus dedicated atomic structure studies profit most from the method. The feature of intrinsic time resolution is unique, and further atomic lifetime and decay rate measurements on selected cases would be very welcome, because they test the atomic structure calculations used in the models of the corona spectrum in a way that is different from just the level energies which are available from spectra. However, for statistically meaningful studies at high spectral resolution, of multiply charged heavy ions of astrophysical interest, the performance parameters are demanding, and the remaining heavy-ion accelerator facilities for moderate to medium high charge state ions may be too limited in ion beam current. In contrast, the line-rich spectra of low-charge state ions of heavy elements, which pervade the UV, visible, and near infrared spectra of astrophysical objects, claim a need for relatively small ion accelerators, nowadays often combined with laser spectroscopic techniques, for many years to come.

Similarly, ions in relatively low charge states should keep tabletop EBITs busy on behest of astrophysicists as well as plasma physicists working on divertors and other wall elements of fusion test reactors. However, fast measurements with small instruments, which are very helpful for plasma diagnostics, will not do for the analysis of complex spectra. The recent range extension of one of the Livermore high-resolution spectrographs bodes well for future measurements in further sections of the EUV spectrum of $\mathrm{Fe}$ as represented by the data in figure 2, because it offers a significantly higher spectral resolving power on ions in an electron density environment that among terrestrial laboratories is closest to the solar corona.

\section{Acknowledgment}

Part of this work was performed under the auspices of the U.S. Department of Energy by Lawrence Livermore National Laboratory under Contract DE-AC52-07NA27344.

\section{References}

1. J.E. Mack, J.R. Stehn, and B. Edlén. J. Opt. Soc. Am. 22, 245 (1932).

2. B. Edlén. Zeits. Astrophysik 22, 30 (1943).

3. A.H. Gabriel, B.C. Fawcett, and C. Jordan. Nature 206, 390 (1965).

4. B.C. Fawcett, A.H. Gabriel, W.G. Griffin, B.B. Jones, and R. Wilson. Nature 200, 1303 (1963).

5. B.C. Fawcett and A.H. Gabriel. Astrophys. J. 141, 343 (1965).

6. B.C. Fawcett. At. Data Nucl. Data Tab. 16, 135 (1975).

7. B.C. Fawcett. At. Data Nucl. Data Tab. 22, 473 (1978).

8. A. Kramida, Yu. Ralchenko, J. Reader, and NIST ASD team (2012). NIST Atomic Spectra Database (ver. 5.0), (Online). Available: http://physics.nist.gov/asd [2012, October 3]. National Institute of Standards and Technology, Gaithersburg, MD, U.S.A.

9. G. Del Zanna, K.P. Dere, P.R. Young, E. Landi, and H.E. Mason, Astron. Astrophys. 582, 56 (2015).

10. V. Domingo, B. Fleck, and A.I. Poland. Sol. Phys. 162, 1 (1995).

11. J.L. Culhane, L.K. Harra, A.M. James, et al. Sol. Phys. 243, 19 (2007). 
12. L. Kay. Phys. Lett. 536 (1963).

13. S. Bashkin and A.B. Meinel. Astrophys. J. 139413 (1964).

14. S. Bashkin. Nucl. Instrum. Meth. 2888 (1964).

15. S. Bashkin. Science 1481047 (1965).

16. E. Träbert. in "Accelerator-based Atomic Physics Techniques and Applications", (Edited by S M Shafroth and J C Austin) (Washington: Am. Inst. Phys.) (1997) p. 567

17. E. Träbert. Phys. Scr. 78, 038103 (2008).

18. P. Beiersdorfer. Can. J. Phys. 86, 1-10 (2008).

19. E. Träbert, H. Winter, P.H. Heckmann, H. v. Buttlar. Nucl. Instrum. Meth. 135, 353 (1976).

20. E. Träbert, Phys. Scr. T8, 112 (1984).

21. K. Shima, N. Kuno, and M. Yamanouchi. Phys. Rev. A 40, 3557 (1989).

22. K. Shima, N. Kuno, M. Yamanouchi, and H. Tawara. At. Data Nucl. Data Tab. 51, 173 (1992).

23. E. Träbert. Z. Phys. D 2, 213 (1986).

24. E. Träbert, R. Hutton, and I. Martinson. Mon. Not. R. astron. Soc. 227 27p 1987.

25. E. Träbert, P.H. Heckmann, R. Hutton, and I. Martinson. J. Opt. Soc. Am. B 521731988.

26. E. Träbert. Mon. Not. R. Astron. Soc. 2973991998.

27. C. Jupén, R.C. Isler, and E. Träbert. Mon. Not. R. Astron. Soc. 264627 (1993).

28. M.A. Levine, R.E. Marrs, J.N. Bardsley, P. Beiersdorfer, C.L. Bennett, M.H. Chen, T. Cowan, D. Dietrich, J. R. Henderson, D.A. Knapp, A. Osterheld, B.M. Penetrante, M.B. Schneider, and J. H. Scofield. Nucl. Instrum. Meth. B 43, 431-440 (1989).

29. D.A. Knapp, R.E. Marrs, S.R. Elliott, E.W. Magee, and R. Zasadzinski. Nucl. Instrum. Meth. Phys. Res. A 334, 305-312 (1993).

30. R.E. Marrs, P. Beiersdorfer, and D.H. Schneider. Physics Today 47, issue 10, 27-34 (1994).

31. S.B. Utter, P. Beiersdorfer, J.R. Crespo López-Urrutia, and K. Widmann. Nucl. Instrum. Meth. Phys. Res. A 428, 276 (1999).

32. S.B. Utter, G.V. Brown, P. Beiersdorfer, E.J. Clothiaux, and N.K. Podder. Rev. Sci. Instrum. 70, 284 (1999).

33. J.K. Lepson, P. Beiersdorfer, G.V. Brown, S.M. Kahn, D.A. Liedahl, C.W. Mauche, and S.B. Utter. Rev. Mex. Astron. Astrophys. (Serie de Conferencias) 9, 137 (2000).

34. J.K. Lepson, P. Beiersdorfer, G.V. Brown, D.A. Liedahl, S.B. Utter, N.S. Brickhouse, A.K. Dupree, J.S. Kaastra, R. Mewe, and S.M. Kahn. Astrophys. J. 578, 648 (2002).

35. E. Träbert, P. Beiersdorfer, E.H. Pinnington, S.B. Utter. M.J. Vilkas, and Y. Ishikawa. J. Phys.: Conf. Ser. 58, 93 (2007).

36. E. Träbert, P. Beiersdorfer, N.S. Brickhouse, and L. Golub. Astrophys. J. Suppl. 211, 14 (2014).

37. J.R. Lemen, A.M. Title, D.J. Akin, P.F. Boerner, C. Chou, J.F. Drake, D.W. Duncan, C.G. Edwards, F.M. Friedlaender, G.F. Heyman, N.E. Hurlburt, N.L. Katz, G.D. Kushner, M. Levay, R.W. Lindgren, D.P. Mathur, E.L. McFeaters, S. Mitchell, R.A. Rehse, C.J. Schrijver, L.A. Springer, R.A. Stern, T.D. Tarbell, J.-P. Wuelser, C.J. Wolfson, C. Yanari, J. A. Bookbinder, P.N. Cheimets, D. Caldwell, E.E. Deluca, R. Gates, L. Golub, S. Park, W.A. Podgorski, R.I. Bush, P.H. Scherrer, M.A. Gummin, P. Smith, G. Auker, P. Jerram, P. Pool, R. Soufli, D.L. Windt, S. Beardsley, M. Clapp, J. Lang, and N. Waltham. Sol. Phys. 275, 17 (2012).

38. E. Träbert, P. Beiersdorfer, N.S. Brickhouse, and L. Golub. Astron. Astrophys. 586, A115 (2016).

39. P. Beiersdorfer, E. W. Magee, E. Träbert, H. Chen, J. K. Lepson, M.-F. Gu, and M. Schmidt. Rev. Sci. Instrum. 75, 3723-3726 (2004).

40. P. Beiersdorfer, E. W. Magee, G. V. Brown, N. Hell, E. Träbert, and K. Widmann. Rev. Sci. Instrum. 85, 11E422 (2014).

41. B. O’Dwyer, G. Del Zanna, H.E. Mason, M.A. Weber, and D. Tripathi. Astron. Astrophys. 521, A21 (2010).

42. S. Parenti, B. Schmieder, P. Heinzel, and L. Golub. Astrophys. J. 754, 66 (2012).

43. E. Träbert, P. Beiersdorfer, N.S. Brickhouse, and L. Golub. Astrophys. J. Suppl. 215, 6 (2014).

44. P. Beiersdorfer, E. Träbert, J.K. Lepson, N.S. Brickhouse, and L. Golub. Astrophys. J. 788, 25 (2014). 


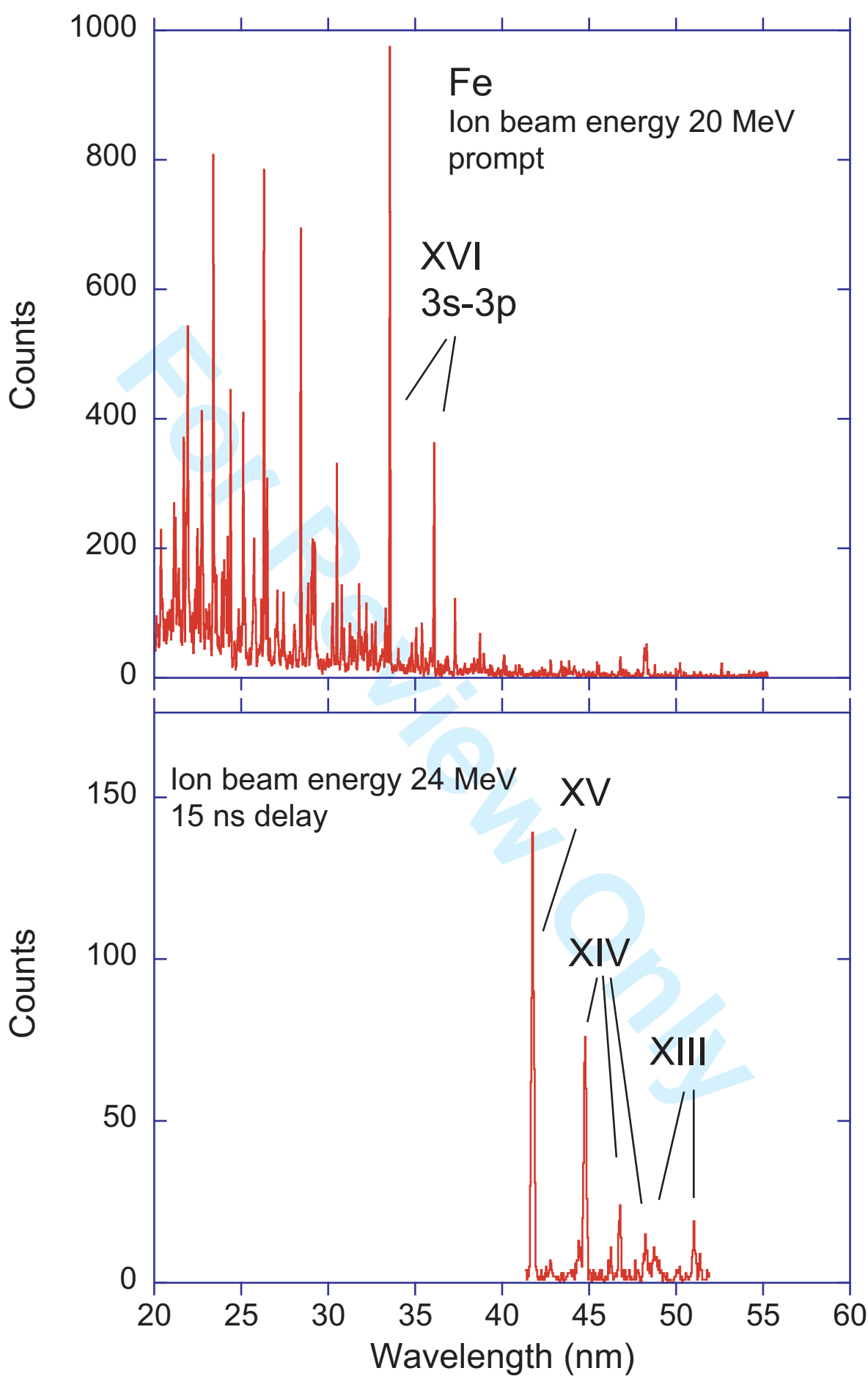

Figure 1. Beam-foil spectra of Fe. The upper spectrum has been recorded close to the exciter foil ('prompt' spectrum). The lower spectrum has been recorded at a distance of about $175 \mathrm{~mm}$ from the foil ('delayed' spectrum), and it is dominated by intercombination transitions). The two spectra have been recorded with different spectrometer slit widths and signal normalization. Under the conditions of the upper experiment, the signal corresponding to the lower spectrum disappears in the "grass".

NRC Canada 


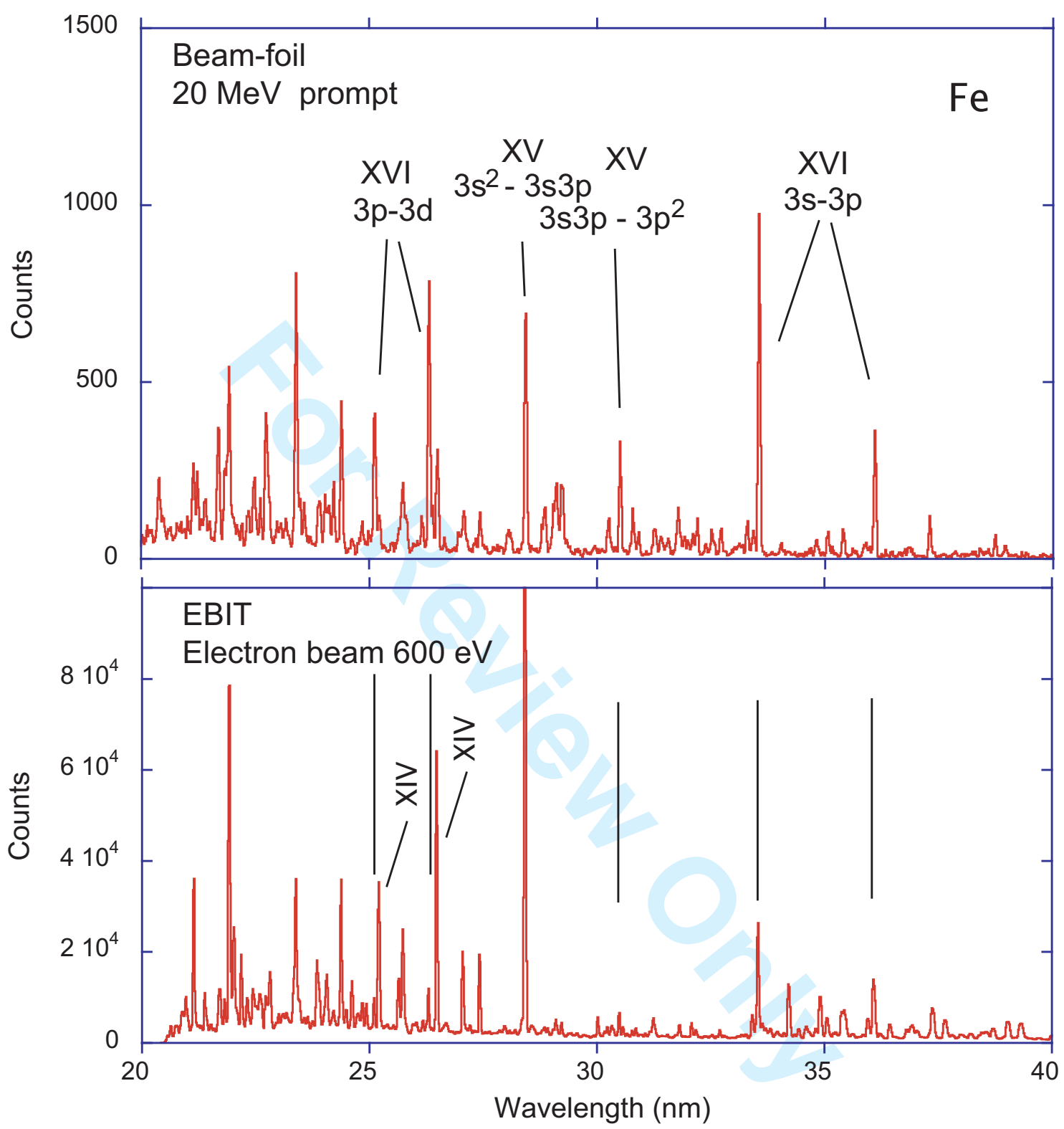

Figure 2. Fe spectra of ions excited by beam-foil interaction (top) and by electron impact in an electron beam ion trap (bottom). Note that the line of highest intensity in the lower spectrum is truncated in favor of a better visibility of the weaker lines. The vertical black markers connect similar lines of Fe XVI and Fe XV in both spectra that are discussed in the text. 